\title{
Reflets
}

Revue ontaroise d'intervention sociale et communautaire

\section{Francophone à Thunder Bay : une activiste sociale parle}

\section{Jocelyne Paquette}

Volume 1, numéro 2, automne 1995

La santé communautaire en Ontario français : défis et espoirs

URI : https://id.erudit.org/iderudit/026090ar

DOI : https://doi.org/10.7202/026090ar

Aller au sommaire du numéro

Éditeur(s)

Reflets : Revue ontaroise d'intervention sociale et communautaire

ISSN

1203-4576 (imprimé)

1712-8498 (numérique)

Découvrir la revue

Citer cet article

Paquette, J. (1995). Francophone à Thunder Bay : une activiste sociale parle. Reflets, 1(2), 232-236. https://doi.org/10.7202/026090ar

Tous droits réservés (C) Reflets : Revue ontaroise d'intervention sociale et communautaire, 1995
Ce document est protégé par la loi sur le droit d'auteur. L'utilisation des services d'Érudit (y compris la reproduction) est assujettie à sa politique d'utilisation que vous pouvez consulter en ligne.

https://apropos.erudit.org/fr/usagers/politique-dutilisation/ 


\section{Francophone à Thunder Bay:}

\section{une activiste sociale parle}

\section{Jocelyne Paquette}

R echerchiste, Thunder B ay

$\mathrm{M}$ a mère est une Acadienne du N ouveau-Brunswick et mon père est un Franco- $O$ ntarien d' $O$ ttawa. J'ai étudié avec les «Soeurs de la Sagesse» jusqu'à l'âge de 14 ans. M on école secondaire était anglaise et j'ai quitté à l'âge de 17 ans. Je ne suis pas retournée aux études avant 1980. En tant que Franco- $O$ ntarienne vivant à Thunder Bay depuis 1991, je ne comprends que trop bien l'effet de l'isolement linguistique. Ce genre d'isolement n'est pas toujours évident, surtout pour ceux et cellesqui font partie du groupe linguistique majoritaire. N éanmoins, les questions de droits linguistiques, en lien avec l'accès des femmes aux services et au soutien, doivent être sérieusement abordées. La source de mon analyse vient directement de mon expérience de travail au sein d'organisations féminines anglophones et francophones aux niveaux municipal, régional et national.

Ville d'un peu plus de 100000 habitants, Thunder Bay est dans tous les sens du mot la métropole du N ord-ouest de I'O ntario. Q ue vous soyez à la recherche d'activités culturelles ou récréatives ou que vous ayez un rendez-vous avec un médecin ou un dentiste, vous vous rendez àT hunder Bay. LesA utochtones dans cette communauté constituent $15 \%$ de la population, et Thunder Bay est fière d'avoir la plus importante communauté finlandaise à l'extérieur de la Finlande.

En 1990, la ville s'est désignée unilingue anglophone même si les Francophones constituaient à ce moment-là 3,1 \% de la 
population (Statistique C anada 1991). L'agent des langues officielles a quitté la ville en 1992. Les problèmes engendrés par ce départ nous ont laissés vulnérables lorsque le Secrétariat d'État du C anada (aujourd'hui le Patrimoine canadien) a décidé que la communauté pouvait avoir accès à des services à Sudbury. A près tout, il n'y a pas suffisamment de Francophones àT hunder Bay! Suite à l'abandon du poste d'agent, la ville ne pouvait plus agir comme point de service pour la communauté francophone du $\mathrm{N}$ ord-ouest de l'O ntario. La situation a alerté la communauté et nous nous sommes mobilisés pour exercer des pressions sur le gouvernement afin qu'il agisse. En 1993, la ville deT hunder Bay et le district comptaient $4 \%$ de Francophones. Encore une fois, le besoin d'un agent ou d'une agente pour la communauté était perçu comme prioritaire. N ous sommes en mars 1995 et le poste doit être comblé bientôt. C e n'est pas surprenant que nous nous sentions négligés et visés.

La communauté francophone du $\mathrm{N}$ ord-ouest est privée de services dans une ville qui ne soutient ni ne tolère la langue française. Allez à la banque et dites «bonjour» au commis. Vous entendrez souvent des soupirs et verrez les gens se retourner, froncer les sourcils. Le conducteur d'autobus peut vous dire de ne pas parler en françai s: "Y ou don't do that here inT hunder B ay». Le résultat: vous vous cachez dans votre langue. Vous faites vos emplettes, traitez avec le gouvernement, tout cela dans la langue majoritaire. Les années passent, vous utilisez de moins en moinsle français et finissez éventuellement par avoir honte d'essayer. $\mathrm{N}$ ous sommes une communauté d'êtres invisibles, souvent isolés les uns des autres. N ous n'avons ni signe distinctif ni attribut particulier qui indique notre langue. En tant que Francophones, nous vivons toujours avec les pressions d'être réduits au silence.

L'activiste qui vit dans cette communauté peut s'attendre à faire face à beaucoup de résistance, à peu d'appui, et finalement, à l'isolement qui survient à travailler seule.

À T hunder Bay, l'Accueil francophone lutte pour les droits des Francophones en matière de services sociaux et de santé, d'appui et d'information. L'Accueil est le seul service d'interprétation et de traduction accessible aux membres de la communauté 
francophone qui ont besoin de tels services. Comme l'une des employées de l'Accueil le dit si bien: «es services d'accompagnement deviennent davantage des services de défense et de revendication alors que le patient ou la patiente sont rarement informés des options possibles. Connaître vos options et vosdroits en tant que membre de la communauté influencera la façon dont vous prendrez des décisions. L'avenir des soins de santé dans cette province et dans ce pays se décide maintenant et l'Accueil aide de façon unique pour voir à ce que les Francophones du $\mathrm{N}$ ordouest de l'O ntario soient entendus. L'Accueil est une présence visible pour une minorité invisible. Son soutien et ses efforts continus dans la lutte pour les droits linguistiques des Francophones demeure une priorité et un engagement.

Comme activiste, on se trouve souvent à marcher sur la corde raide. La position de Francophone symbolique en est une que nous connaissons bien. Les organisations disent servir les Francophones uniquement parce qu'ils peuvent dire «on en a une sur notre conseil d'administration!». C ette déclaration démontre peu de soutien ou de service.Vous attendez, vous continuez de faire votre travail, mais éventuellement vous atteignez le point où vous pensez exploser. $O \mathrm{n}$ entend dire: «N ous ne savons pas pourquoi les femmes francophones ne viennent pass. L'inclusion, I'acceptation et le respect des diversités sont absents de la discussion. Personne ne représente une communauté entière et personne ne devrait le faire. En tant que féministes, nous devons lutter contre un modèle d'accès exclusif, tenace et limité qui a depuis longtemps été établi dans le système patriarcal.

Et quelle est l'histoire de la femmme francophone dans les grandes distances du $\mathrm{N}$ ord- $\mathrm{O}$ uest? Le féminisme demeure une anomalie pour de nombreuses Francophones. $N$ ous reconnaissons que nous occupons une position subordonnée et souvent marginale dans le système patriarcal mais en même temps nous soutenons, défendons et maintenons cette structure. Pour illustrer ce point, au cours d'une réunion d'un groupe de femmes francophones, j'ai entendu les commentaires suivants sur l'histoire d'une victime de viol. L'extrait de film que nous venions de visionner avait décrit le viol d'une femme par le fiancé de sa 
meilleure amie. La phrase, «elle n'aurait pas dû être là de toute façon!» a été dite de trois façons différentes. Finalement, c'était la victime qui devait être blâmée. Enfermées dans une société patriarcale stricte, en tant que femmes francophones, nous avons peu de possibilités de refaire le moule. $\mathrm{N}$ otre direction, notre formation et notre éducation ont été étroitement liées à l'Église et à la maison. C'est à l'intérieur de ces limites que nous devons essayer de trouver notre propre place dans la société. N os expériences, comme celles de nombreuses femmes au $\mathrm{C}$ anada, nous amènent à nous sentir paralysées et angoissées devant la violence.

Comme femmes francophones, nous continuons à nous éduquer sur nos réalités. M ême si le $C$ omité canadien sur la violence faite aux femmes (1993) a commis des erreurs méthodologiques et a peu fait pour aider à comprendre l'inclusion, les statistiques ont démontré ce que les femmes de ce pays savaient déjà: la violence fait partie de notre quotidien. Toutefois, peu ou pas de services sont offerts aux femmes d'ici. D 'abord, bien qu'il existe plus d'une dizaine de lignes téléphoniques d'urgence (1-800) dans le nord-ouest de l'O ntario, aucune n'est désignée pour offrir des services en français. D e plus, les maisons de transition et les maisons d'hébergement ne sont pas obligées d'avoir une employée bilingue. Finalement, il est difficile d'accéder à la documentation francophone sur la violence et peu de ressources ont été créées spécifiquement pour lesfemmes francophones. L'expression voulant «qu'il y a toujours quelque chose de perdu dans la traduction» est une réalité puisque la plupart du matériel que nous recevons ne reflète ni notre culture, ni notre expérience.

Pourtant, il y a encore de l'espoir. Les femmes francophones sont en train de faire face à leur double isolement et commencent à discuter des problèmes qui affectent leur vie. Le Comité des femmes francophones du N ord-ouest de l'O ntario a complété, en mai 1994, un sondage intitulé «S'informer, c'est prévenir» concernant la violence contre les femmes francophones dans le nord-ouest de l'O ntario. Les femmes francophones se sont fait entendre en participant à ce sondage avec un taux de réponse de $20 \%$. C omme le sondage a été distribué à 1200 femmes francophones du $\mathrm{N}$ ord-ouest de l'O ntario, le taux élevé de réponses 
reflète un engagement et un désir de contribuer à des changements sociaux positifs. Les commentaires ont clairement identifié le besoin d'accès à des services telle une ligne téléphonique d'urgence gratuite. Lesfemmes ont identifié des sentiments d'isolement et des inquiétudes face aux grandes distances qui doivent être parcourues pour se rendre aux centresqui offrent des services. $M$ ême si de nombreux rapports semblables ont déjà été faits, peu ont rejoint la communauté francophone avec un tel succès. Ce combat en est un que nous devons gagner pour toutes les femmes.

Pour conclure, je voudrais dire que la langue n'est pas limitée aux mots écrits et prononcés. La langue fait partie de notre culture, elle nous définit et nous renouvelle tout au long de notre vie. Ce n'est qu'en atteignant la quarantaine que je me suis découverte. Le féminisme et un besoin d'influences linguistiques et culturelles se sont suivis comme un coup de foudre. Comme de nombreuses femmes francophones, je suis revenue à ce que j'avais perdu, un sentiment d'identité et des liens avec une culture qui demeure forte et vibrante. $C$ 'est cet engagement et ce dynamisme qui réuniront nos expériences et nous aideront à faire les changements nécessaires pour célébrer la diversité, élargir notre compréhension et bâtir des communautés fortes, sûres et équitables pour tous leurs membres.

Les femmes francophones du nord-ouest de I'O ntario ainsi que moi-même vous remercions et vous demanderons votre appui afin que nous puissions obtenir notre ligne d'urgence gratuite. C'est une question de solidarité qui a un impact direct sur la qualité de vie des femmes du $\mathrm{N}$ ord.

\section{Bibliographie}

Le comité sur la violence faite aux femmes (1993). U n nouvel horiz on: É liminer la violence - A tteindre I'égalité, $O$ ttawa, ministère desA pprovisionnements et Services $C$ anada.

Statistique C anada (1991) «G roupes minoritaires, chiffres de la population selon la première langue officielle», recensement de la population 1991.

Statistique $\mathrm{C}$ anada « $\mathrm{F}$ rançais comme réponse simple et multiple à la question sur la langue maternelle», recensement de la population, $R$ ecensement pour l'O ntario. 